\title{
Automatic Spinal Deformity Detection Based on Neural Network
}

\author{
Hyoungseop Kim¹, Seiji Ishikawa ${ }^{1}$, Marzuki Khalid², Yoshinori Otsuka ${ }^{3}$, \\ Hisashi Shimizu ${ }^{4}$, Yasuhiro Nakada ${ }^{4}$, Takasi Shinomiya ${ }^{5}$, and Max A. Viergever6 \\ ${ }^{1}$ Faculty of Engineering, Kyushu Institute of Technology, 1-1, Sensui-cho, Tobataku, \\ Kitakyushu, 804-8550 Japan \\ kim@cntl.kyutech.ac.jp \\ ${ }^{2}$ Center for AI and Robotics, Universiti Teknologi Malaysia \\ ${ }^{3}$ National Sanatorium Chiba Higashi Hospital Japan \\ ${ }^{4}$ Chiba Health Care Association Japan \\ ${ }^{5}$ Nikon Digital Technologies Co., Ltd Japan \\ ${ }^{6}$ Image Science Institute, University Hospital Utrecht The Netherlands
}

\begin{abstract}
We propose a technique for automatic spinal deformity detection method from moiré topographic images. Normally the moiré stripes show a symmetric pattern, as a human body is almost symmetric. According to the progress of the deformity of a spine, asymmetry becomes larger. Numerical representation of the degree of asymmetry is therefore useful in evaluating the deformity. Displacement of local centroids is evaluated statistically between the left-hand side and the right-hand side regions of the moiré images with respect to the extracted middle line. The degree of the displacement learned by a neural network employing the back propagation algorithm. An experiment was performed employing 1,200 real moiré images (600 normal and 600 abnormal) and $89 \%$ of the images were classified correctly by the NN.
\end{abstract}

\section{Introduction}

In recent years various imaging techniques have been introduced into medical fields. Accordingly many related image processing techniques have been reported [1-2] in medical imaging fields. We proposed some techniques [3-6] for automatic judging of spinal deformity using approximate symmetry analysis from moiré topographic images [7]. The present technique for automatic spinal deformity detection aims at being introduced in the primary screening stage mainly performed at schools to release doctors from tough work of visual inspection of a large number of moiré images of subjects backs and to realize objective judgment. Those suspicious cases found in this primary screening are going to be sent further to X-ray examination for detailed inspection.

Spinal deformity is a disease mainly suffered by teenagers during their growth stage particularly from fifth year on element school to second year on middle school. There are many causes of spinal deformity, but all of them are unknown. The most common type is termed "idiopathic" that show $80 \%$ of the spinal deformity. If one has spinal deformity, his spine is crooked and the ribs may stick out more on one side 
than the other side. When one afflicted with spinal deformity, his/her spine often deforms in the shape of letter ' $S$ ' or ' $C$ '. In severe case, when one becomes a spinal deformity, it associated with pain and he requires surgical treatment. In order to check the presence of spinal deformity in its early stage, orthopedists have traditionally performed on children a painless examination called a forward-bending test. In forward-bending test, mainly medical doctor checks to see if one shoulder is lower than the other. But this test is neither reproductive nor objective. Moreover the inspection takes much time when applied to medical examination in schools. To overcome these difficulties, a moiré method [8] has been proposed which takes moiré topographic images of human backs and checks symmetry/asymmetry of the moiré patterns in a two-dimensional (2-D) way. Invention of the moiré method largely raised efficiency of the school scoliosis examination. One of the main reasons why the moiré method has gained its popularity among doctors is that symmetry/asymmetry of a human back can clearly be observed by its moiré pattern. It is, however, medical doctors who examine the moiré images and visual examination of a large amount of moiré images collected from elementary as well as junior high schools. This causes exhaustion of doctors and therefore leads to misjudgment. Therefore realization of automated scoliosis inspection based on the moire images has long been desired among orthopedists.

Automating judgment of spinal deformity by computer has been reported [9-14] employing moiré images of human backs. Despite these efforts, their approaches did not succeed, because of the difficulty of image processing in extracting the moire stripes exactly. Thus they did not reach to the stage of classification experiments employing real data.

In this paper, we will apply a new method which the authors have developed [3-6], for automatic spinal deformity detection on moiré topographic images of human backs. The proposed technique is separated into four main steps. In the first place, once the original moire image is fed into computer, the middle line of the subject's back is extracted on the moiré image employing the approximate symmetry analysis. Regions of interests (ROIs) are then automatically selected on the moiré image from its upper part to the lower part. Displacement of local centroids is evaluated statistically between the left-hand side and the right-hand side regions of the moiré images with respect to the extracted middle line. In the final place, normal and abnormal cases learned by a neural network employing the back propagation algorithm.

\section{Extraction of the Middle Line and the Degree of Asymmetry}

Symmetry is one of the important structural properties of shapes in computer visions. There are many shapes having approximate symmetry, i.e., human faces, and symmetric shapes with asymmetric texture. All of these are can be understood to have bilateral symmetry. The human back is not exactly symmetric, even if the subject does not suffer from spinal deformity. Normally the moiré stripes show symmetric patterns on the human body is almost symmetric. According to the progress of the deformity of a spine, asymmetry of the pattern becomes larger. Numerical representation of the degree of asymmetry may therefore be useful in the evaluating the spinal deformity. In order to analyze such shapes with approximate symmetry, 
some techniques are proposed [15-16]. Ishikawa et al. [17] proposed a technique for detecting symmetry axes on an approximately symmetric shape and applied it to extracting the middle line of a human back from its moiré image.

\subsection{Definition of the Middle Line}

The middle line of the subject's back is extracted on the given moiré image employing the approximate symmetry analysis [17]. The middle line is extracted in the following way.

Let a digital image be denoted by $f(x, y)$ where $x$ and $y$ are bounded positive integers. The $(p+q)$ th-order moment of $f(x, y)$ is defined by

$$
m_{p q}=\sum \sum_{(x, y) \in R} x^{p} y^{q} f(x, y)
$$

where $R$ is a specified region. By calculating the centroid $\left(x_{c}, y_{c}\right)$ by

$$
\left(x_{c}, y_{c}\right)=\left(\frac{m_{10}}{m_{00}}, \frac{m_{01}}{m_{00}}\right) \text {. }
$$

From the given an original image, its reflected image is represented by $f^{r}(x, y)$, $(x, y) \in R^{r}$. The reflection image is superposed onto the original image by parallel translation $c \equiv\left(c_{x}, c_{y}\right)$ and rotation $\theta$ to find the best match. In other words, transformation $T^{*}$ is searched such as the followings holds;

$$
\left.\begin{array}{c}
e_{T}=\sum_{(x, y) \in R \cup R^{r}}\left|\frac{f(x, y)-T f^{r}(x, y)}{S}\right| \\
e^{*} \equiv e_{T^{*}}=\min _{T} e_{T}
\end{array}\right\}
$$

where

$$
T=\left[\begin{array}{ccc}
\cos \theta & \sin \theta & c_{x} \\
-\sin \theta & \cos \theta & c_{y} \\
0 & 0 & 1
\end{array}\right]
$$

and $S=n\left\{R \cup R^{f}\right\}$. Here the number of the elements of a set $A$ is denoted by $n(\mathrm{~A})$. Note that the following geometrical restriction is taken into account with respect to $c_{x}$, $c_{y}$ and $\theta$;

$$
c_{y}=c_{x} \tan \frac{\theta}{2} .
$$

In this paper, we assume the $\theta=0$ in eq.(4), because the moiré images are captured normally straight using positioner so that their middle lines keep vertical. 


\subsection{Extracting of Regions of Interest}

In the next stage, we extract the region of interests from a given moiré image. The region of interests is extracted in the following way.

Let us denote a moiré image of a human back by $I(x, y)$. The origin $O$ of the $x y$ coordinate system is located at the lower left corner of the image. The ranges of the coordinates are $0 \leqq x \leqq x_{e}$ and $0 \leqq x \leqq y_{e}$. The middle line is defined in the first place on $I(x, y)$. Since the moiré pattern of a human back usually exhibits asymmetry, an approximate symmetry axis is extracted from $I(x, y)$ and the axis is regarded as the middle line of the back. The middle line is located at $x=m$.

The region of interests denoted by $R$ is defined on $I(x, y)$ in the following way. Image $I(x, y)$ is binarized and histogram of the binarized pixels onto $x$-axis is calculated. The locations having the minimum frequency on the histogram are searched within $0 \leqq x \leqq m$ and $m \leqq x \leqq x_{e}$, and two such locations, $x=x_{0}$ and $x=x_{1}$, that are the nearest to the middle line are chosen from the respective ranges. The area $R$ excludes arms of the subject and takes subject's physical dimensions into account. The regions of interests (ROIs) are automatically selected by this processing.

\subsection{Extracting of Asymmetric Degree}

Figure 1 illustrates the ROIs and calculation area on the human backs. Within the region $R$ and at a certain position $y=i$, two rectangle areas are defined, as shown in Figure 1, at symmetric locations with respect to the middle line $x=m$. The width $a$ of the rectangle area is defined by

$$
a=\min \{m-l, r-m\} .
$$

On the other hand, height of the area is defined empirically. The degree of asymmetry $D$ is calculated by following way.

Let us denote the rectangle areas of the left-hand side and right-hand side at $y=i$ by $A_{i}^{l}$ and $A_{i}^{r}$, respectively. Here $i=1,2, \cdots, N$. The centroids of $A_{i}^{l}$ and $A_{i}^{r}$ are denoted by $G_{l}\left(x_{l}, y_{l}\right)$ and $G_{r}\left(x_{r}, y_{r}\right)$, respectively. The centroid $G_{l}\left(x_{l}, y_{l}\right)$ is reflected with respect to the middle line $x=m$ into the region $A_{i}^{r}$ and denoted by $G_{l}^{*}\left(x_{l}{ }^{*}, y_{l}{ }^{*}\right)$. The distance $D$ between $G_{l}^{*}\left(x_{l}^{*}, y_{l}^{*}\right)$ and $G_{r}\left(x_{r}, y_{r}\right)$ is calculated by

$$
D=\sqrt{\left(x_{l}^{*}-x_{r}\right)^{2}+\left(y_{l}^{*}-y_{r}\right)^{2}} .
$$

The mean $\mu$ and the standard deviation $\sigma$ of the values $D(i=1,2, \cdots, N)$ are employed as the features representing the degree of asymmetry of the moiré image in $R$. They are obtained from

$$
\left.\begin{array}{l}
\mu=\frac{1}{N} \sum_{i=1}^{N} D \\
\sigma=\sqrt{\frac{1}{N} \sum_{i=1}^{N}(D-\mu)^{2}}
\end{array}\right\}
$$




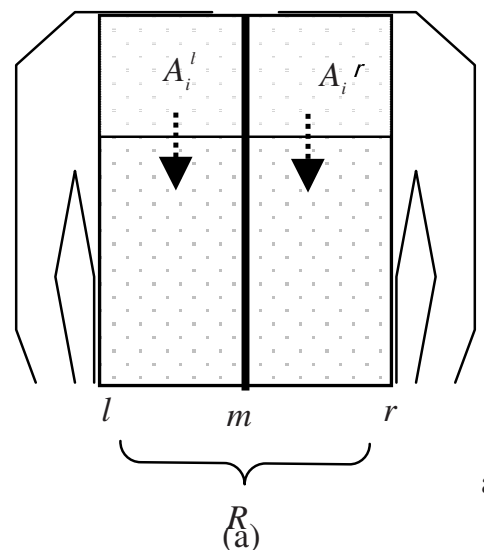

(a)

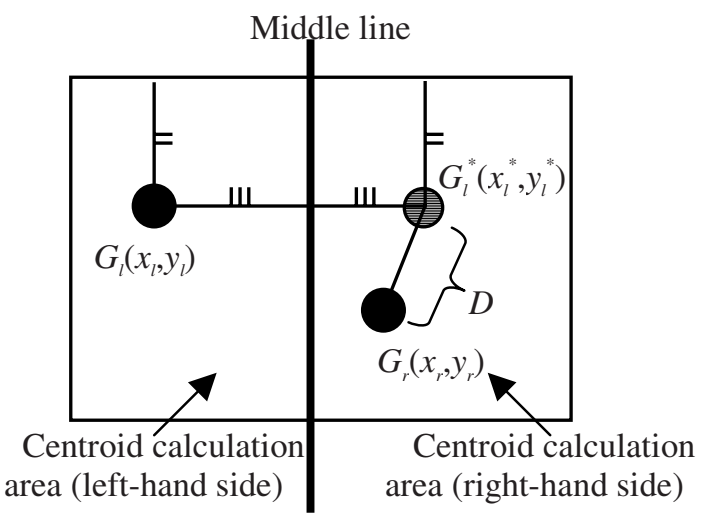

(b)

Fig. 1. ROIs and calculation area: (a) Region of Interests; (b) Calculation area

\section{Classification by NN}

Neural network (NN) is used to describe a computer model assumption of the biological brain. It consists of a set of interconnected simple processing units, which combine to output a signal to solve a certain problem based on the input signals it received. The interconnected simple processing units have adjustable giants that are slowly adjusted through iterations influenced by the input-output patterns given to the NN.

Neural networks have been proven in many researches as having a good discriminant property, which means excellent for pattern classification. Neural networks accept numerical inputs and provide classification based on these inputs by segregating the inputs. This application to automatic spinal deformity can provide the necessary inputs, which are numerical in nature to the neural network. The implementation is very easy, as we only need to train the neural network based on past data or even current data. In this case we find that the backpropagation algorithm which is a supervised learning neural network to be very appropriate to solve this classification application of automatic spinal deformity detection.

A direct application would be to take the 2 feature vectors from the left-hand side and right-hand side rectangle areas ( $\mu$ and $\sigma$ in eq.(8)) and apply them to train a NN employing back propagation algorithm for automatic spinal deformity detection.

Employed NN is consists of 3 layers, which included two input layers, three hidden layers and two output layers for training. Finally, unknown moiré images are discriminated as normal or abnormal case automatically. Figure 2 illustrates the employing neural network system for classification. 


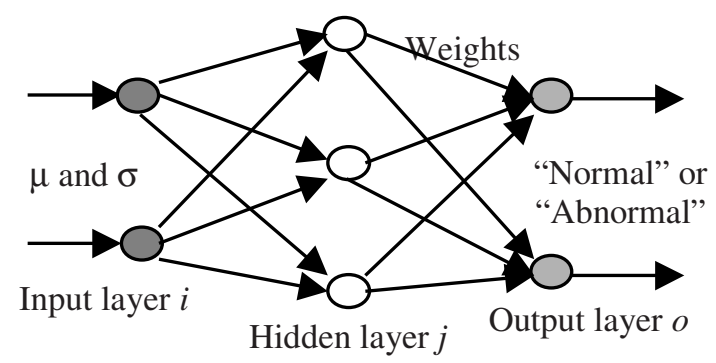

Fig. 2. Employed Neural Network for classification

\section{Experimental Results}

According to the above mentioned procedure, experiment was done employing 1200 (600 normal cases and 600 abnormal cases) real moiré images. The moiré topographic images fed into an EWS through a personal computer. The image size is $256 \times 256$ pixels with 256 gray levels and they are provided by FD or MO disk. The program is written in $\mathrm{C}$ language. In the neural networks for learning 800 (400 normal cases and 400 abnormal cases) moiré images are employed, remaining 400 (200 normal cases and 200 abnormal cases) moiré images are used for classification. In average, classification rate of $89 \%$ was achieved. The processing time of a singe moire image is 2.6 second in average on a Pentium III $(1 \mathrm{GHz})$ personal computer running FreeBSD.

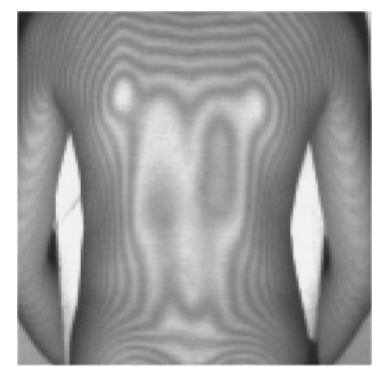

(a)

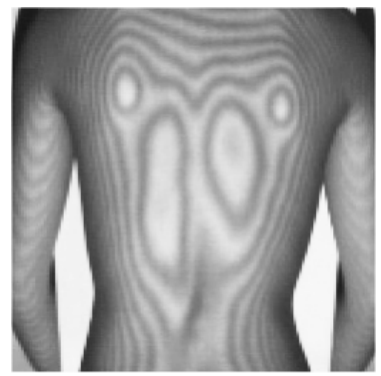

(b)

Fig. 3. Examples of misclassification: (a) A normal case classified into abnormal; (b) An abnormal case classified into normal

\section{Discussions and Conclusion}

In this paper, we propose a technique for analyzing a moiré image of a human back in a 2-D way in order to automate the primary screening of spinal deformity detection based on neural network. This approach seems promising compared with existent other attempts which analyze moiré images in a 3-D way. In fact, the present technique achieved the classification rate of $89 \%$ in the experiment employing 1200 
real moiré images, whereas other techniques based on 3-D analysis have not even performed such experiments. The present technique offers simpler 2-D image processing. This also can be reduces computation time.

Some other features were as well employed for the experiments, such as difference of areas [17], inclination of the principal axes [3], etc., between the left part and the right part of the moiré images. Further development and improvement of the features describing asymmetry might result in higher classification rate in the present automatic inspection.

Figure 3 illustrates examples of misclassification. In Figure 3(a), a normal case is classified into abnormal, whereas an abnormal case is classified into normal in (b). In Figure 3(a), gray values subtly differ in the vicinity of an edge particularly on the shoulder part. All of the 17 misclassified cases are found asymmetry of moiré patterns and yet extracted local centroids spread on the image almost in a symmetric way. This is because gray values distribution in the rectangle regions unfortunately affected symmetrically when the local centroids were calculated. To escape from this difficulty, some other asymmetry features independent to local centroids displacement might be taken into account in conjunction with it. The issue remains for further study.

From this database consist of 200 normal moiré topographic images and 200 abnormal moiré topographic images, the results were a sensitivity of 0.92 at a specificity of 0.88 . Furthermore, False Positive Fraction (FP) of the 0.12 and True Positive Fraction (TP) of the 0.92 were achieved under the Receiver Operating Characteristic (ROC) analysis.

The classification rate of $89 \%$ is achieved in the experiment. In more detail, $87 \%$ of normal cases were recognized correctly and $92 \%$ of abnormal cases were recognized correctly. In this point, the present technique misclassifies normal cases as abnormal at a higher rate than abnormal cases as normal, which should be strictly avoided.

\section{References}

1. Han, C.Y., Lin, K.N., William, G.W, Robert, M.M., David T.P.: "Knowledge-based image analysis for automated boundary extraction of transesophageal echocardiographic leftventricular images", IEEE Trans. on Medical Imaging, 10, 4, 602-610(1991).

2. Michael, W., Jeffrey, L.D., Jonathan, S.L.: "Interventional magnetic resonance imaging: Concept and applications in neuroradiology", Japanese Society of Medical Imaging Tech., 17, 6, 658-667(1999).

3. Kim, H.S., Ueno, H., Ishikawa, S., Otsuka, Y.: "Recognizing asymmetric moiré patterns for human spinal deformity detection", Proceedings of Korea Automatic Control Conference, pp.568-571(1997).

4. Ishikawa, S., Eguchi, T., Yamaguchi, T., Kim, H.S., Otsuka, Y.: "Judging spinal deformity by two characteristic axes on a human back", Proceedings of Korea Automatic Control Conference, pp.438-441 (1996).

5. Kim, H.S., Motoie, M., Ishikawa, S., Ohtsuka, Y., Shimizu, H.: "Spinal deformity detection based on 2-D evaluation of asymmetry of moiré patterns of the human back", Proceedings of 1999 International Technical Conference on Circuits/Systems, Computers and Communications, 673-676(1999). 
6. Kim, H.S., Ishikawa, S., Ohtsuka, Y., Shimizu, H., Sinomiya, T., Viergever, M.A.: "Automatic scoliosis detection based on local centroids evaluation on moiré topographic images of human backs", IEEE Trans. Med. Imag., TMI-20, 12, 1314-1320(2001).

7. Takasaki, H.: "Moiré topography", Appl. Opt., 9, 1457(1970).

8. Ohtsuka, Y., Shinoto, A., Inoue, S.: "Mass school screening for early detection of scoliosis by use of moiré topography camera and low dose X-ray imaging” , Clinical Orthopaedic Surgery, 14, 10, 973-984 (1979). (in Japanese)

9. Idesawa, M., Yatagai, T., Soma, T.: "Scanning moiré method and automatic measurement of 3-D shapes” , Appl. Opt., 16, 2152-2162 (1977).

10. Batouche, M.: “A knowledge based system for diagnosing spinal deformations: Moiré pattern analysis and interpretation” , Proc. 11 Int. Conf. Pattern Recogn., 591-594 (1992).

11. Adair, I.V., Wijk, M.C., Armstrong, G.W.D.: "Moiré topography in scoliosis screening", Clin. Orthop., 129, 165(1977).

12. Wilner, S.: "Moiré topography for the diagnosis and documentation of scoliosis", Acta Orthop. Scand., 50, 295(1979).

13. Roger, R.E., Stokes, I.E., et al.: "Monitoring adolescent idiopathic scoliosis with moiré fringe photography", Engineering in Medicine, 8, 119(1979).

14. Ishikawa, S., Takagami, S., Kato, K., Ohtsuka, Y. "Analyzing deformity of human backs based on the 3-D topographic reconstruction from moiré images" , Proc. '95 Korea Automat. Control Conf., 244-247 (1995).

15. Minovic, P., Ishikawa, S., Kato, K.: “Three-dimensional symmetry identification, Part I: Theory” , Memoir of the Kyushu Institute of Technology, 21, 1-17 (1992).

16. Minovic, P., Ishikawa, S., Kato, K.: "Symmetry identification of a 3-D object represented by octree” , IEEE Trans. Patt. Anal. Machine Intell., PAMI-15, 5, 507-514 (1993).

17. Ishikawa, S., Kosaka, H., Kato, K., Ohtsuka, Y.: "A method of analyzing a shape with potential symmetry and its application to detecting spinal deformity" , Comput. Vision, Virtual Reality, Robotics in Med., 465-470, Springer (1995). 